\title{
Central Configurations of the Five-Body Problem with Equal Masses
}

\author{
October 31, 2018 \\ Tsung-Lin Lee \\ Department of Mathematics \\ Michigan State University \\ East Lansing, MI 48824, USA \\ leetsung@msu.edu \\ Manuele Santoprete \\ Department of Mathematics \\ Wilfrid Laurier University \\ 75 University Avenue West, \\ Waterloo, ON, Canada, N2L 3C5. \\ msantopr@wlu.ca
}

\begin{abstract}
In this paper we present a complete classification of the isolated central configurations of the five-body problem with equal masses. This is accomplished by using the polyhedral homotopy method to approximate all the isolated solutions of the Albouy-Chenciner equations. The existence of exact solutions, in a neighborhood of the approximated ones, is then verified using the Krawczyk method. Although the Albouy-Chenciner equations for the five-body problem are huge, it is possible to solve them in a reasonable amount of time.
\end{abstract}

\section{Introduction}

The Newtonian $n$-body problem is the study of the dynamics of $n$ point particles with masses $m_{i} \in \mathbb{R}^{+}$and positions $q_{i} \in \mathbb{R}^{d}(i=1, \ldots, n)$, moving according to Newton's laws of motion:

$$
m_{j} \ddot{q}_{j}=\sum_{i \neq j} \frac{m_{i} m_{j}\left(q_{i}-q_{j}\right)}{r_{i j}^{3}} \quad 1 \leq j \leq n
$$

where $r_{i j}=\left\|q_{i}-q_{j}\right\|$ is the distance between $q_{i}$ and $q_{j}$.

In the Newtonian $n$-body problem, the simplest possible motions are such that the configuration is constant up to rotations and scaling, and each body describes a Keplerian orbit. Only some special configurations of particles are allowed in such motions. 
Wintner called them central configurations. A configuration $\left(q_{1}, \ldots, q_{n}\right)$ is called a central configuration if and only if there exists a $\lambda \in \mathbb{R}$ such that

$$
\lambda\left(q_{j}-q_{G}\right)=\sum_{i \neq j} \frac{m_{i}\left(q_{i}-q_{j}\right)}{r_{i j}^{3}} \quad 1 \leq j \leq n
$$

where $q_{G}=\sum_{i} m_{i} q_{i} / \sum_{i} m_{i}$ is the center of mass. Equations (2) are invariant under rotations, dilatations and translations on the plane. Two central configurations are considered equivalent if they are related by these symmetry operations.

The question of the existence and classification of central configuration is a fascinating problem that dates back to the 18th century. In 1767, Euler discovered the collinear c.c.'s. In 1772 Lagrange proved that, for any three arbitrary masses, the equilateral triangle is a central configuration.

For the collinear $n$-body problem an exact count of the central configurations of $n$ bodies was found by Moulton [36] (see also [43] for a modern proof). There is a unique collinear relative equilibrium for any ordering of the masses so there are $n ! / 2$ collinear equivalence classes.

The number of planar central configurations of the $n$-body problem for an arbitrary given set of positive masses, has been established only for $n=3$ : there are always five relative equilibria. Two of these are Lagrange's equilateral triangles and the other three are collinear c.c. discovered by Euler. Already in the four-body problem there is sufficient complexity to prevent a complete classification of the non-collinear relative equilibria. In fact, an exact count is known only for the equal masses case [1, 2] and for certain cases where some of the masses are assumed sufficiently small [52, 45]. Some partial result in the four-body problem with some equal masses are given in [30, 38, 6].

Even the finiteness of the central configurations is a very difficult question. This conjecture was proposed by Chazy [10] and Wintner [51] and was listed by Smale as problem number 6 on his list of problems for this century [44]. The finiteness problem was settled by Albouy [1, 2] for the case of four equal masses and by Hampton and Moeckel [16] for the general four body problem.

Aside from these fundamental results very little else is known in terms of the classification of planar c.c.'s for $n \geq 4$. Strictly spatial five-body central configurations are analogous in some ways to the planar four-body case, and a classification of the symmetric ones is given in [18]. Planar five-body central configurations present new challenges and there are very few results in this area.

In this paper, we present all the isolated central configurations of the five-body problem with equal masses. Such central configurations have been obtained by finding all the isolated solutions of the Albouy-Chenciner equations (see Section 2 below, and [5, 16]) for this problem. Numerical explorations of the problem have been conducted by Moeckel [33] and Ferrario [11]. They obtained isolated solutions using root-finding routines with a random starting point. However, unfortunately, those numerical experiments do not guarantee that there are no other solutions, and do not ensure that approximate solutions correspond to exact solutions. Our approach is different, and it is based on the homotopy continuation method. This is a technique that was proposed to find all the isolated solutions of polynomial systems [17, 26]. We used the polyhedral homotopy method to find approximate solutions because it needs to trace fewer 
curves than other homotopy methods.

Already for four bodies the Albouy-Chenciner equations are quite large - 12 equations in 12 variables with total degree 2,985,984. Moreover, even when the polyhedral homotopy method is applied, 82,593 curves need to be traced. Experiments running on a $3.2 \mathrm{GHz}$ CPU machine show that the polynomial system solver HOM4PS-2.0 obtains all the isolated solutions of the four-body problem in 10 minutes, while other similar software solvers require more than 10 hours to perform the same task. Considering that, in the five-body case, the number of curves that need to be traced reaches 439, 690,761 we use the kernel of HOM4PS-2.0 to find approximated isolated solutions. We then verify the existence of an exact solution in a small neighborhood of the approximate one by using the Matlab toolbox INTLAB, an implementation of the interval Krawczyk method [19, 35].

Note that, while the polyhedral homotopy method finds all the isolated complex solutions, this does not exclude the existence of positive-dimensional components of the variety defined by the Albouy-Chenciner system in the algebraic torus $\left(\mathbb{C}^{*}\right)^{20}=$ $(\mathbb{C} \backslash\{0\})^{20}$. Hence, at least in principle, there could still conceivably be undiscovered "continua" of positive real solutions. The fact that this is a nontrivial possibility is illustrated by Roberts' example of a continuum of real solutions in the Newtonian 5problem with one negative mass [40].

The paper is organized as follows. The derivation of the Albouy-Chenciner equations is shown in Section 2 In Section 3 we introduce the homotopy continuation method and several related software packages. The computation results are described in Section 4 In Section 5 all the central configurations of the five-body problem with equal masses are presented.

\section{Albouy-Chenciner Equations}

The Albouy-Chenciner equations are algebraic equations satisfied by the mutual distances $r_{i j}$ of every central configuration [5, 16]. For convenience of the reader, in this section, we present a beautiful derivation of the Albouy-Chenciner equations due to Hampton and Moeckel [16].

Multiplying the $j$-th equation of (2) by $m_{j}$ and summing gives $m q_{G}=\sum_{j=1}^{n} m_{j} q_{j}$, where $m=\sum_{j=1}^{n} m_{j}$. Then, after setting $\lambda=m \lambda^{\prime}$, the central configuration equations become:

$$
\sum_{i=1}^{n} m_{i} S_{i j}\left(q_{i}-q_{j}\right)=0, \quad 1 \leq j \leq n,
$$

where

$$
S_{i j}=\frac{1}{r_{i j}^{3}}+\lambda^{\prime} \quad(i \neq j), \quad S_{i i}=0 .
$$

Introducing a $d \times n$ configuration matrix $Q$ whose columns are the position vectors $q_{i}$ we can write equation (2) as

$$
Q A=0,
$$


where $A$ is the $n \times n$ matrix with entries:

$$
A_{i j}=m_{i} S_{i j} \quad(i \neq j) \quad A_{j j}=-\sum_{i \neq j} A_{i j}
$$

The matrix $Q$ can be viewed as representing a linear map from a space of dimension $n$ ( $n$ being the number of bodies) to the physical space in $\mathbb{R}^{d}$ where the points are located.

The most important point of the derivation is to replace $Q$ by some quantity which is invariant under rotations and translations of the position vectors $q_{i}$ in $\mathbb{R}^{d}$. Of course any such quantity can be expressed as a function of the mutual distances $r_{i j}$.

Translation of all the positions $q_{i}$ by a vector $u \in \mathbb{R}^{d}$ transforms $Q$ to $Q+u L$ where $L$ is the $1 \times n$ vector whose components are all 1 . Thus translation invariance can be achieved by restricting the linear map defined by $Q$ to the plane $P=\left\{v \in \mathbb{R}^{n}: L v=\right.$ $\left.v_{1}+\ldots+v_{n}=0\right\}$. Since the sum of the elements of each column of $A$ is zero it follows that $A: \mathbb{R}^{n} \rightarrow P$ and it restricts to $A: P \rightarrow P$. So $Q A$ can be viewed as a linear map of $P$ into $\mathbb{R}^{d}$.

Rotation invariance is obtained by passing to Gram matrices. For any configuration matrix $Q$, the Gram matrix $G=Q^{t} Q$ is the $n \times n$ matrix whose entries are the Euclidean inner products $q_{i} \cdot q_{j}$. G is obviously rotation invariant. To maintain translation invariance, one can view $G$ as representing a symmetric bilinear form $\beta(v, w)=v^{t} G w$ on $P$. The form $\beta$ on $P$ determines and is determined by the mutual distances $r_{i j}$. To see this, note that for any constant $k_{i}$, adding the vector $k_{i} L$ to the row $i$ of $G$ and the vector $k_{i} L^{t}$ to the column $i$ produces a new matrix representing $\beta$. Choosing $k_{i}=-\frac{1}{2}\left|q_{i}\right|^{2}$ shows that $\beta$ is represented by the matrix $B$ whose entries are

$$
B_{i j}=q_{i} \cdot q_{j}-\frac{1}{2}\left|q_{i}\right|^{2}-\frac{1}{2}\left|q_{j}\right|^{2}=-\frac{1}{2} r_{i j}^{2}
$$

Multiplying both sides of (5) by $Q^{t}$ gives $G A=0$. The matrix $G A$ can be viewed as representing a (non-symmetric) bilinear form on $P$, in which case it is permissible to replace $G$ by $B$. Taking the symmetric part gives the Albouy-Chenciner equations for central configurations:

$$
B A+A^{t} B=0 .
$$

Let $e_{i}$ denote the standard basis vectors in $\mathbb{R}^{n}$ and define $e_{i j}=e_{i}-e_{j}$. Then 8 is equivalent to the equations

$$
e_{i j}^{t}\left(B A+A^{t} B\right) e_{i j}=0 \quad 1 \leq i<j \leq n
$$

To see this, let $\gamma(v, w)=v^{t} C w$ be the symmetric bilinear form on $P$ associated to the matrix $C=B A+A^{t} B$. Then (9) means that $\gamma\left(e_{i j}, e_{i j}\right)=0$ for $1 \leq i<j \leq n$. To show that $\gamma=0$ it suffices to show that $\gamma$ vanishes on the basis $e_{1 i}, 2 \leq i \leq n$ of $P$. By the polarization identity

$$
2 \gamma\left(e_{1 i}, e_{1 j}\right)=\gamma\left(e_{i j}, e_{i j}\right)-\gamma\left(e_{1 i}, e_{1 i}\right)-\gamma\left(e_{1 j}, e_{1 j}\right),
$$

this follows from 9 . 
Equations 9 provide $\left(\begin{array}{l}n \\ 2\end{array}\right)$ constraints on the $\left(\begin{array}{l}n \\ 2\end{array}\right)$ mutual distances $r_{i j}$ of a central

configuration. Conversely, it can be shown that if the quantities $r_{i j}$ are mutual distances of some configuration in $\mathbb{R}^{d}$, and if they satisfy (9), then the configuration is central. Note that these equations determine the central configurations in all dimensions at once.

To find the equation explicitly, note that

$$
\gamma\left(e_{i j}, e_{i j}\right)=2 e_{i j}^{t} B A e_{i j}=2\left((B A)_{i i}+(B A)_{j j}-(B A)_{i j}-(B A)_{j i}\right),
$$

where $(B A)_{i j}$ denotes the entries of the matrix $B A$. From (6) and (7) we find

$$
\sum_{k=1}^{n} m_{k}\left[S_{i k}\left(r_{j k}^{2}-r_{i k}^{2}-r_{i j}^{2}\right)+S_{j k}\left(r_{i k}^{2}-r_{j k}^{2}-r_{i j}^{2}\right)\right]=0
$$

for $1 \leq i<j \leq n$, where $S_{i k}$ and $S_{j k}$ are given by (4).

Finally, to eliminate the dilation symmetry, we choose $\lambda^{\prime}=-1$.

\section{The homotopy continuation method}

Let $P(x)=0$ be a system of $n$ polynomial equations in $n$ unknowns. Denoting $P(x)=$ $\left(p_{1}(x), \ldots, p_{n}(x)\right)$ and $x=\left(x_{1}, \ldots, x_{n}\right)$, we want to find all the isolated solutions of

$$
P(x)=\left\{\begin{array}{c}
p_{1}\left(x_{1}, \ldots, x_{n}\right)=0 \\
\vdots \\
p_{n}\left(x_{1}, \ldots, x_{n}\right)=0
\end{array}\right.
$$

in $\mathbb{C}^{n}$. The classical homotopy continuation method [26] for solving $P(x)=0$ is to find a trivial system $Q(x)=\left(q_{1}(x), \ldots, q_{n}(x)\right)$ and then follow the solution curves in the real variable $t$ from $t=0$ to $t=1$ which make up the solution set of

$$
H(x, t)=(1-t) c Q(x)+t P(x)=0 \text { with generic } c \in \mathbb{C} \backslash\{0\} .
$$

More precisely, all isolated solutions of $P(x)=0$ can be found if the system $Q(x)=$ 0 , known as the start system, is chosen properly to satisfy the following three properties:

- Property 0. The solutions of the start system $Q(x)=0$ are known;

- Property 1 . The solution set of $H(x, t)=0$ for $0 \leq t \leq 1$ consists of a finite number of smooth paths, and each of them can be parametrized by $t$ in $[0,1)$;

- Property 2. Every isolated solution of $H(x, 1)=P(x)=0$ can be reached by some path originating at $t=0$, that is, the path starts from a solution of the start system $H(x, 0)=Q(x)=0$.

A typical choice of a start system $Q(x)=0$ satisfying Property 0-2 is

$$
Q(x)=\left\{\begin{aligned}
q_{1}\left(x_{1}, \ldots, x_{n}\right) & =a_{1} x_{1}^{d_{1}}-b_{1} \\
\vdots & \\
q_{n}\left(x_{1}, \ldots, x_{n}\right) & =a_{n} x_{n}^{d_{n}}-b_{n}
\end{aligned}\right.
$$




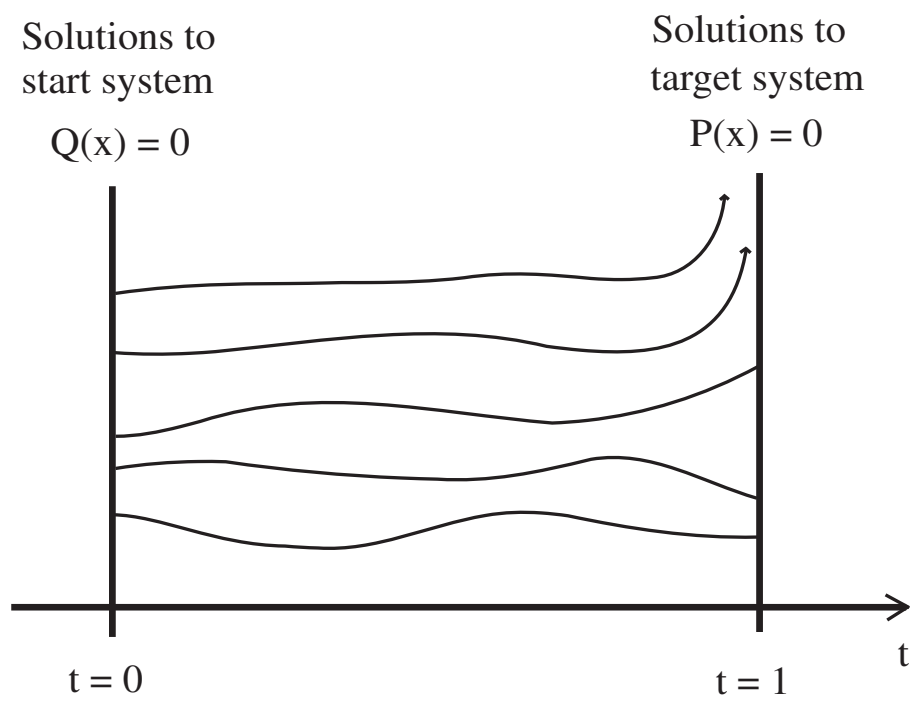

Figure 1: The solution set of classical homotopy.

where $d_{1}, \cdots, d_{n}$ are the degrees of polynomials $p_{1}(x), \ldots, p_{n}(x)$ respectively, and $a_{j}, b_{j}, j=1, \cdots, n$ are random complex numbers [24, 25, 26]. The solutions of such start system $Q(x)=0$ can be explicitly obtained and the total number of solutions is $d=d_{1} \times d_{2} \times \cdots \times d_{n}$, which is known as the total degree or the Bézout number of the original polynomial system $P(x)=0$. We may then find all the isolated solutions of $P(x)=0$ by following the total degree number of paths originated from solutions of the start system $Q(x)=0$. But, a great majority of the polynomial systems arising in applications have fewer than, and in some cases only a small fraction of, $d=d_{1} \times$ $\cdots \times d_{n}$ isolated zeros. We call such a system deficient. In this case, many of the $d_{1} \times \cdots \times d_{n}$ paths will diverge to infinity as $t \rightarrow 1$ (see Figure 1), and those paths become extraneous, causing highly wasteful computations.

In the middle of 1990's, a major computational breakthrough emerged in solving deficient polynomial systems. The new method, called the polyhedral homotopy continuation method [17], takes a great advantage of the combinatorial root count, called mixed volume, in the Bernshteín's theorem [7], which generally provides a much tighter bound for the number of isolated zeros of a polynomial system in the algebraic torus $\left(\mathbb{C}^{*}\right)^{n}=(\mathbb{C} \backslash\{0\})^{n}$. When this method is employed, the number of homotopy paths that need to be traced agrees with the mixed volume of the polynomial system. Therefore, when the mixed volume of a polynomial system is far less than its total degree, the method will greatly reduce the extraneous paths and thereby considerably limit the wasteful computations.

However, this method involves a sometimes costly computation - the mixed cell computation. This mixed cell computation can become very costly for large polynomial systems. In 2005, a software package, MixedVol [12], emerged which led the existing codes for the mixed volume computation by a great margin in speed. However, soon after MixedVol was published, Mizutani, Takeda, and Kojima [32] developed a more 
efficient algorithm which considerably outperformed MixedVol. Most recently Lee and Li [22] embedded the novel idea of dynamic enumeration of mixed cells of Mizutani, Takeda, and Kojima [32] into MixedVol and a new code, MixedVol-2.0, was produced which regained the lead by a substantial margin.

The software package HOM4PS [12], developed over the years by the group led by T. Y. Li at Michigan State University, implemented the polyhedral homotopy continuation method for solving polynomial systems. The code was widely considered as the most efficient polynomial systems solver. Recently many new curves tracing techniques and mixed cell computation algorithms, such as MixedVol-2.0 mentioned above, have been included in HOM4PS-2.0. By incorporating these new algorithms HOM4PS-2.0 is (as reported in [21]), in general, at least one order of magnitude faster than HOM4PS, as well as from one to three orders of magnitude faster than PHCpack [47], PHoM [13] and Bertini [8]. Consequently, some large size polynomial systems can be solved in a reasonable number of hours. However, as the size of the polynomial system becomes larger, we need more computing resources to solve the system more efficiently. A natural way to attain more computing resources is to parallelize the homotopy continuation method which seems to be naturally parallel in the sense that each isolated zero is computed independently of the others. The parallel version of HOM4PS-2.0, HOM4PS-2.0para, has also been developed. Its excellent scalability in numerical results are reported in [27].

\section{Computing central configurations}

The Albouy-Chenciner equations for the five-body problem with equal masses form a polynomial system consisting of 20 equations in 20 variables. For finding all the isolated solutions in complex field, the polyhedral homotopy method needs to trace $439,690,761$ curves. In order to trace so many curves efficiently, we use the subroutines in the MPI library (message passing interface [37]) to distribute data over multiple CPUs for parallel computation and we use the kernel of HOM4PS-2.0 to construct polyhedral homotopies and trace curves. Employing 32 Itanium2 1.6 GHz CPUs, the computation is completed in 140 hours. As a result we obtain 101,062,826 solutions in the complex field, 8775 of which are real solutions. Among those real solutions only 258 satisfy the physical condition (i.e. $r_{i j}>0$ for all $1 \leq i<j \leq 5$ ). We assume that any numerical solution with imaginary parts less than the threshold $\theta=10^{-7}$ corresponds to a real solution of the Albouy-Chenciner equations. This is reasonable since the set of real solutions remains the same when the threshold $\theta$ is chosen between $10^{-13}$

and $10^{-3}$. In addition, the residuals of the physical solutions are less than $2 \times 10^{-14}$ and their condition numbers are at most $3.8 \times 10^{2}$, which show that these solutions are numerically reliable.

To guarantee that in a small neighborhood of each numerical solution there is a unique exact physical solution, the interval Krawczyk method [19, 35] is applied for verification. The method is based on the fact: For a smooth function $F: \mathbb{R}^{n} \rightarrow \mathbb{R}^{n}$ and a point $\mathbf{x} \in \mathbb{R}^{n}$, let $[\mathbf{x}]_{r} \subset \mathbb{R}^{n}$ be the interval set centered at $\mathbf{x}$ with radius $r>0$. Namely,

$$
[\mathbf{x}]_{r}=\left\{\mathbf{y} \in \mathbb{R}^{n}:\|\mathbf{y}-\mathbf{x}\|_{\infty} \leq r\right\},
$$


where $\|\cdot\|_{\infty}$ is the infinity norm. Assume the derivative of $F$ at $\mathbf{x}$, denoted by $D F(\mathbf{x})$, is nonsingular, the Krawczyk set of $F$ associated with $[\mathbf{x}]_{r}$ is defined as

$$
K\left(F,[\mathbf{x}]_{r}\right)=\mathbf{x}-D F(\mathbf{x})^{-1} F(\mathbf{x})+\left[I-D F(\mathbf{x})^{-1} D F\left([\mathbf{x}]_{r}\right)\right]\left([\mathbf{x}]_{r}-\mathbf{x}\right) .
$$

If the Krawczyk set is contained in the interior of $[\mathbf{x}]_{r}$, then there exists a unique zero of $F$ in $[\mathbf{x}]_{r}$.

The task of verification is implemented by using the interval arithmetic in INTLAB (INTerval LABoratory) [41], developed by Siegfried M. Rump at Hamburg University of Technology, Germany. In this implementation each numerical solution $\mathbf{x}$ is taken as the midpoint of the interval set $[\mathbf{x}]_{r}$ with radius $r=10^{-8}$. The numerical solutions obtained by running HOM4PS-2.0 and the results of verification by using INTLAB are available for download at http://hom4ps.math.msu . edu/Nbody.htm.

\section{Central Configurations of the 5 body problem}

In this section we present all the central configurations of the five-body problem with equal masses classified according to their dimensions. The dimension of the configuration was deduced by using Cayley-Menger determinants [48]. For instance the CayleyMenger determinant for the volume $V$ of the four-dimensional simplex is

$$
-9216 V^{2}=\left|\begin{array}{cccccc}
0 & 1 & 1 & 1 & 1 & 1 \\
1 & 0 & r_{12}^{2} & r_{13}^{2} & r_{14}^{2} & r_{15}^{2} \\
1 & r_{12}^{2} & 0 & r_{23}^{2} & r_{24}^{2} & r_{25}^{2} \\
1 & r_{13}^{2} & r_{23}^{2} & 0 & r_{34}^{2} & r_{35}^{2} \\
1 & r_{14}^{2} & r_{24}^{2} & r_{34}^{2} & 0 & r_{45}^{2} \\
1 & r_{15}^{2} & r_{25}^{2} & r_{35}^{2} & r_{45}^{2} & 0
\end{array}\right| .
$$

Configurations of dimension three or less were found by setting the determinant above to zero. Configurations of dimension two or less were found by setting to zero the determinant above plus the following determinants

$$
\left|\begin{array}{ccccc}
0 & 1 & 1 & 1 & 1 \\
1 & 0 & r_{12}^{2} & r_{13}^{2} & r_{14}^{2} \\
1 & r_{12}^{2} & 0 & r_{23}^{2} & r_{24}^{2} \\
1 & r_{13}^{2} & r_{23}^{2} & 0 & r_{34}^{2} \\
1 & r_{14}^{2} & r_{24}^{2} & r_{34}^{2} & 0
\end{array}\right|,\left|\begin{array}{ccccc}
0 & 1 & 1 & 1 & 1 \\
1 & 0 & r_{12}^{2} & r_{13}^{2} & r_{15}^{2} \\
1 & r_{12}^{2} & 0 & r_{23}^{2} & r_{25}^{2} \\
1 & r_{13}^{2} & r_{23}^{2} & 0 & r_{35}^{2} \\
1 & r_{14}^{2} & r_{24}^{2} & r_{35}^{2} & 0
\end{array}\right|,\left|\begin{array}{ccccc}
0 & 1 & 1 & 1 & 1 \\
1 & 0 & r_{23}^{2} & r_{24}^{2} & r_{25}^{2} \\
1 & r_{23}^{2} & 0 & r_{34}^{2} & r_{35}^{2} \\
1 & r_{24}^{2} & r_{34}^{2} & 0 & r_{45}^{2} \\
1 & r_{25}^{2} & r_{35}^{2} & r_{45}^{2} & 0
\end{array}\right|,
$$

where, for example, the first of the three determinants above is the Cayley-Menger determinants for the tetrahedron formed by the masses $m_{1}, m_{2}, m_{3}$ and $m_{4}$. One-dimensional configurations were obtained by setting to zero, in addition to the four Cayley-Menger determinants above, the ten Cayley-Menger determinants corresponding to all the possible triangles formed by a subset of three of the five masses. One of the ten determinants is

$$
-16 A^{2}=\left|\begin{array}{cccc}
0 & 1 & 1 & 1 \\
1 & 0 & r_{12}^{2} & r_{13}^{2} \\
1 & r_{12}^{2} & 0 & r_{23}^{2} \\
1 & r_{13}^{2} & r_{23}^{2} & 0
\end{array}\right|
$$




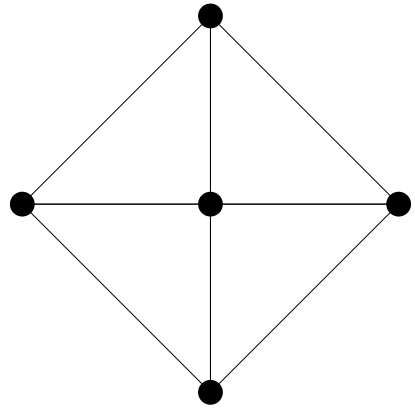

(a)

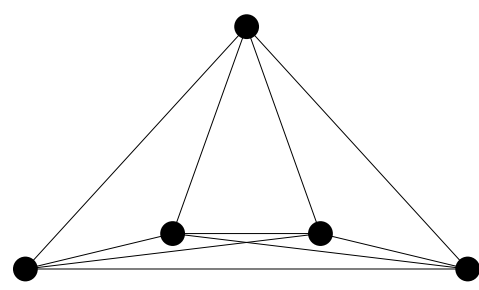

(c)

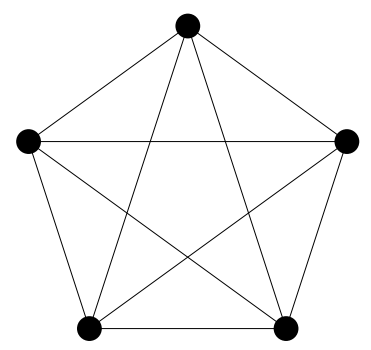

(b)

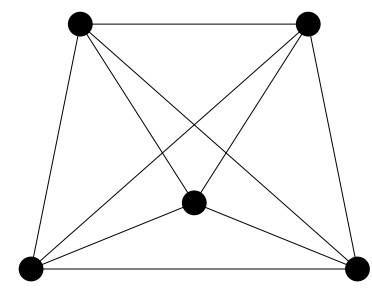

(d)

Figure 2: Planar central configurations of the five body problem with equal masses.

where $A$ is the area for a plane triangle with side lengths $r_{12}, r_{13}$ and $r_{23}$.

\subsection{Collinear and four-dimensional central configurations}

A complete count of the collinear central configurations has been known for almost one century. In 1910 Moulton [36] proved that there is a unique central configuration corresponding to each possible ordering of $n$ masses along a line, and thus there are $n ! / 2$ collinear central configurations. In 1970 Smale used tools of the theory of dynamical systems to give a modern proof of Moulton's result.

Our computations show, in agreement with Moulton's result, that there are $60=$ $5 ! / 2$ solutions of the Albouy-Chenciner equations.

The four-dimensional central configurations of five bodies are also well understood. Saari [42] proved that the regular $n-1$ dimensional simplex is a central configuration of $n$ bodies for any value of the masses. In particular the case $n=4$ has been well known over a century [23]. The fact that the tetrahedron is the unique spatial central configuration of four bodies was proved by Pizzetti [39]. We find one 4-dimensional solution of the Albouy-Chenciner equations that, as we expected, is a regular 4-dimensional 
simplex.

\subsection{Planar Central Configurations}

There are very few results concerning the planar five-body problem. Part of the work on the planar five body problem dates back to more than 50 years ago and is due to Williams [49],[50]. More recently, in 2005, Hampton [15] found new examples of (symmetric) central configurations in the planar five-body problem. Llibre and Mello [28] and Llibre, Mello, and Perez-Chavela [29] found some further central configurations using the same techniques employed by Hampton. Not much more is known in the planar five-body problem

It has been known for a long time that, in the equal mass case, the regular pentagon and the square with one mass at each of its vertices and with the fifth mass at its center are central configurations [49].

In the case of equal masses we find 147 planar solutions of the Albouy-Chenciner equations. Of these, 15 form a square with a particle in the middle (Figure 2(a)), 12 form a regular pentagon (Figure 2 (b)), 60 form an isosceles triangle with the remaining particles in the convex hull of the first three (Figure2 (c)), and 60 form an isosceles trapezoid with a mass in the convex hull of the first four (Figure2(d)). The configurations of Figure 2(c) and Figure2 (d) have been found numerically in 1989 by Moeckel [33] and rediscovered by Ferrario [11]. Note that in [49] Williams erroneously states that a concave pentagon with three masses at the vertices of a pentagon and the remaining two in its interior cannot form central configurations. Our results (see figure Figure 2 (c)) show that such statement is incorrect.

It is interesting to remark that the number of solutions obtained can be explained using their symmetry. In the case of the configuration of Figure (2 a)) the four masses at the vertices of a square and one at the center are subject to an action of the group $S_{5}$ (symmetric group on 5 letters) permuting the masses and hence the set of mutual distances $r_{i j}$. But, the set of mutual distances $R$ for any particular numbering of the masses has an isotropy subgroup that is equal to the symmetries of the square (the dihedral group $D_{4}$ of order 8). Hence the set of $S_{5}$-orbits of configurations ( that is equal to the number of solutions of the Albouy-Chenciner equations) of this type should have $120 / 8=15$ elements. Similarly, the pentagon configurations in Figure (2 a)) have the dihedral group $D_{5}$ as isotropy subgroup and there are $120 / 10=12$ orbits. The configurations in Figure (2 (a)) and (2 (b)) have $C_{2}$ (i.e. the cyclic group of order 2) as isotropy subgroup and, in this case, there are $120 / 2=60$ orbits.

Another interesting fact is that, as in the four-body problem with equal masses [1], all the planar central configuration have an axis of symmetry that contains at least one mass.

\subsection{Spatial Central Configurations}

In 1908 Brehm [9] found some symmetric spatial central configurations of five bodies. Almost one century later Kotsireas and Lazard [18] used linear algebra and Gröbner bases to classify symmetric spatial central configurations of five bodies with equal

masses. They found four distinct classes of central configurations. Kotsireas and 


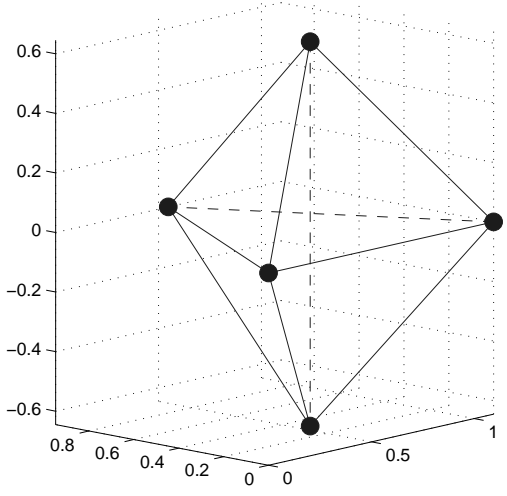

(a)



(b)

Figure 3: Convex spatial central configurations of five bodies with equal masses.

Lazard conjectured that their list of central configurations was complete. Xia proved, for general masses, that there is at least one convex central configuration that is a minimum of the configurational measure [53]. Moeckel used Morse theory, under the assumption of nondegenerate critical points, to find 6 more central configurations of saddle type [34].

We find 50 spatial solutions of the Albouy-Chenciner equations. These correspond to four distinct classes of central configurations (two convex and two concave) that match the ones of Kotsireas and Lazard, thus proving their conjecture to be correct.

The first class has 10 solutions. These consist of an equilateral triangle and two symmetric points on the axis through the barycenter of the triangle that is orthogonal to it (Fig. 3 (a)). The second one contains 15 solutions. These are pyramidal central configurations with a square base (Fig. 3 b)). The third class consists of 5 solutions. These consist of a regular tetrahedron with a point in its barycenter (Fig. 4(a)). The last class contains 20 solutions. These consist of an equilateral triangle and two points lying on the axis through the center of the triangle (Fig. 4(b)).

As in the planar case the number of central configurations can be explained using their symmetry. In the case of the configuration of Figure (3)a)) the masses are subject to an action of the group $S_{5}$. But, the set of mutual distances has the group $D_{3} \times C_{2}$ (of order 12) as isotropy subgroup. Hence the set of $S_{5}$-orbits of configurations of this type should have $120 / 12=10$ elements and thus there are 10 solutions of the Albouy Chenciner equations. The configuration in Figure $(3 \mathrm{~b})$ ) has the dihedral group $D_{4}$ as isotropy subgroup and there are $120 / 8=15$ solutions. The configuration in Figure (4) (a)) has the tetrahedral group $T_{d}$ (that has order 24) as isotropy subgroup and hence there are $120 / 24=5$ solutions. 


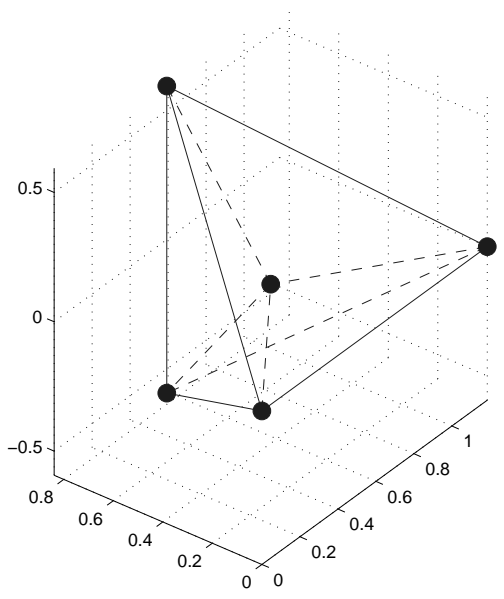

(a)

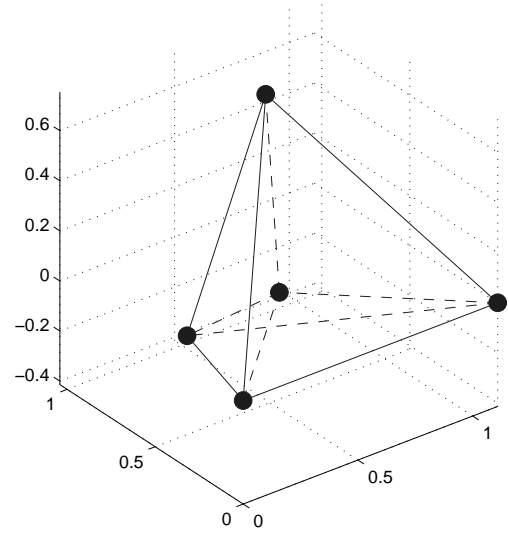

(b)

Figure 4: Concave spatial central configurations of five bodies with equal masses.

The configuration in Figure (4)(b)) has the dihedral group $D_{3}$ as isotropy subgroup and, in this case, there are $120 / 6=20$ solutions.

According to Moeckel [34] the convex central configurations would fit with the Morse theory as follows: 6 saddles plus 9 more $=15,1$ minimum plus 9 more $=10$.

\section{Acknowledgments}

This work was made possible by the facilities of the Shared Hierarchical Academic Research Computing Network (SHARCNET:www.sharcnet.ca) and by INTLAB (INTerval LABoratory) a Matlab toolbox for self-validating algorithms, developed by Siegfried M. Rump at Hamburg University of Technology, Germany. The authors thank Chih-Hsiung Tsai for his assistance in collecting all the complex solutions of the Albouy-Chenciner equations, and Alain Albouy for bringing to our attention unpublished numerical computations by Moekel and Ferrario [11]. Manuele Santoprete was supported by a NSERC Discovery grant.

\section{References}

[1] Albouy A.: Symétrie des configurations centrales de quatre corps. C.R. Acad. Sci Paris. 320, 217-220 (1995)

[2] Albouy A.: The Symmetric Central Configurations of Four Equal Masses, Contemp. Math. V 198, 131-135 (1996) 
[3] Albouy A.: On a Paper of Moeckel on Central Configurations. Reg. Chaotic Dynamics 8, 133-142 (2003)

[4] Albouy A.: Mutual Distances in Celestial Mechanics. preprint (2004)

[5] Albouy A., Chenciner A.: Le probléme des $n$ corps et les distances mutuells. Invent. Math. 131, 151-184 (1998)

[6] Albouy A., Fu Y. and Sun S.: Symmetry of planar four-body convex central configurations. Proc. R. Soc. Lond. Ser. A Math. Phys. Eng. Sci. 464, 1355-1365 (2009)

[7] Bernshteín, D. N.: The number of roots of a system of equations. Functional Analysis and Appl., 9(3), 183-185 (1975)

[8] Bates, D. J., Hauenstein, J. D., Sommese, A. J., Wampler, C. W.: Bertini: Software for Numerical Algebraic Geometry, available at http://www.nd.edu/ sommese/bertini

[9] Brehm, E.: Pertikuläre Integrale des Problems der drei Körper, Dissertation, Berlin (1908)

[10] Chazy J.: Sur Certaines trajectoires du probléme des n corps. Bull. Astron. 35, 321-389 (1918)

[11] Ferrario D. L.: Central configurations, symmetries and fixed points. e-print, math.DS/0204198 (2002).

[12] Gao, T., Li, T. Y., Wu, M.: Algorithm 846: MixedVol: A software package for mixed Volume computation.ACM Transactions on Math. Software, 31(4), 555560 (2005)

[13] Gunji, T., Kim, S., Kojima, M., Takeda, A., Fujisawa, K., Mizutani, T.: PHoM A polyhedral homotopy continuation method. Computing, 73, 57-77 (2004)

[14] Hagihara Y. Celestial Mechanics, Vol. 1. MIT press, Massachusetts (1970)

[15] Hampton M.: Stacked central configurations: new examples in the five-body problem, Nonlinearity 18, 2299-2304 (2005)

[16] Hampton M. and Moeckel R.: Finiteness of Relative Equilibria of the Four-Body Problem, Invent. Math. 163, 289-312 (2006)

[17] Huber, B., Sturmfels, B.: A polyhedral method for solving sparse polynomial systems. Math. of Comp., 64, 1541-1555 (1995)

[18] Kotsireas I., Lazard D.: Central configurations of the 5-body problem with equal masses in three-dimensional space. J. Math. Sci., 108, 1119-1138 (2002)

[19] Krawczyk R.: Newton-Algorithmen zur Bestimmung von Nullstellen mit Fehlerschranken, Computing 4, 187-201 (1969) 
[20] Leandro E.S.G.: Finitness and Bifurcations of some Symmetrical Classes of Central Configurations. Arch. Rational Mech. Anal. 167, 147-177 (2003)

[21] Lee, T. L., Li, T. Y. and Tsai, C. H.: HOM4PS-2.0: A software package for solving polynomial systems by the polyhedral homotopy continuation method. Computing, 83, 109-133 (2008)

[22] Lee, T. L., Li, T. Y.: Mixed volume computation, A revisit. (Submitted).

[23] Lehmann-Filhés R: Ueber zwei Fälle des Vielkörperproblems. Astron. Nachr. 127, 137-143 (1891)

[24] Li, T. Y.: Numerical solution of multivariate polynomial systems by homotopy continuation methods. ACTA Numerica, 399-436 (1997)

[25] Li, T. Y.: Solving polynomial systems by polyhedral homotopies. Taiwan J. of Math., 3251 - 279 (1999)

[26] Li, T. Y.: Solving polynomial systems by the homotopy continuation method. Handbook of numerical analysis, Vol. XI, (pp. 209-304), North-Holland, Amsterdam (2003)

[27] Li, T. Y. and Tsai, C. H.: HOM4PS-2.0para: Parallelization of HOM4PS-2.0 for solving polynomial systems. (Submitted).

[28] Llibre J., Mello L.F.: New central configurations for the planar 5-body problem. Cel. Mech. Dyn. Astr. 100, 141-149 (2008)

[29] Llibre J., Mello L.F. and Perez-Chavela E.: New stacked central configurations for the planar 5-body problem. preprint.

[30] Long Y., Sun. S.: Four-Body Central Configurations with some Equal Masses. Arch. Rational Mech. Anal. 162, 24-44 (2002)

[31] MacMillan W., Bartky W.: Permanent Central Configurations in the Problem of Four Bodies. Trans Amer. Math. Soc. 34, 838-875 (1932)

[32] Mizutani, T., Takeda, A., Kojima, M.: Dynamic enumeration of all mixed cells. Discrete Comput. Geom., 37, 351-367 (2007)

[33] Moeckel R.: Some relative equilibria of N equal masses, preprint (1989)

[34] Moeckel R.: Personal communication.

[35] Moore R. E.: A Test for Existence of Solutions for Non-Linear Systems, SIAM J. Numer. Anal. 4, 611-615 (1977)

[36] Moulton F. R.: The Straight Line Solutions of the Problem of $n$-bodies. Ann. Math. 12, 1-17 (1910)

[37] MPI (message passing interface): http://www-unix.mcs.anl.gov/mpi/ 
[38] Perez-Chavela E., Santoprete M.: Convex four-body central configurations with some equal masses. Arch. Ration. Mech. Anal. 185, 481-494 (2007)

[39] Pizzetti P.: Casi Particolari del Problema dei tre corpi. Rendiconti 13, 17-26 (1904)

[40] Roberts G.: A Continuum of Relative Equilibria in the 5-Body Problem Physica D. 127, 141-145 (1999)

[41] Rump S. M.: INTLAB - INTerval LABoratory. In Tibor Csendes, editor, Developments in Reliable Computing, pages 77-104. Kluwer Academic Publishers, Dordrecht, (1999)

[42] Saari D.: On the role and properties of $n$-body central configurations. Celest. Mech. 21, 9-20 (1980)

[43] Smale S.: Topology and Mechanics II. Inv. Math, 11, 45-64 (1970)

[44] Smale S.: Mathematical Problems for the Next Century. Math. Intell., 20, 7-15 (1998)

[45] Tien F.: Recursion Formulas of Central Configurations, Thesis, University of Minnesota (1993)

[46] Uspensky J.V.: Theory of Equations. McGraw Hill, New York (1948)

[47] Verschelde, J.: Algorithm 795: PHCpack: A general-purpose solver for polynomial systems by homotopy continuation. ACM Trans. Math. Softw., 25, 251-276. Software available at http://www.math.uic.edu/ jan (1999)

[48] Sommerville, D. M. Y.: An Introduction to the Geometry of n Dimensions. Dover, New York, (1958)

[49] Williams W. L.: Permanent configurations in the problem of five bodies. Trans. Am. Math. Soc. 44, 563-579 (1938)

[50] Williams W. L.: A Pentagon Theorem. Am. Math. Mon. 60, 616-617 (1953)

[51] Wintner A.: The Analytical Foundations of Celestial Mechanics. Princeton Math. Series 5. Princeton University Press, Princeton NJ (1941)

[52] Xia, Z.: Central Configurations with Many Small Masses. J. Diff. Eqns., 91, 168179 (1991)

[53] Xia Z.: Convex Central Configurations for the $n$-body Problem. J. Diff. Eqns., 200,185-190 (2004) 